\title{
Detection of Kita-Kyushu Lung Cancer Antigen-1, a Cancer/testis Antigen, in the stomach closer to the cancerous condition.
}

Takashi Fukuyama ( $\sim$ fukuyam@insti.kitasato-u.ac.jp )

Kitasato University: Kitasato Daigaku https://orcid.org/0000-0003-1772-3478

\section{Toshikazu Otsuka}

Kitasato University: Kitasato Daigaku

\section{Nobue Futawatari}

Toho University: Toho Daigaku

Kumiko Tahara

Kitasato University: Kitasato Daigaku

Masaaki Watanabe

Kitasato University: Kitasato Daigaku

Yoshinobu Ichiki

Kitasato University: Kitasato Daigaku

Yatsushi Nishi

Kitasato University: Kitasato Daigaku

Yoshihito Takahashi

Kitasato University: Kitasato Daigaku

Hitoshi Yamazaki

Kitasato University: Kitasato Daigaku

Noritada Kobayashi

Kitasato University: Kitasato Daigaku

Mitsuhiro Kida

Kitasato University: Kitasato Daigaku

Hiroshi Nonoguchi

Kitasato University: Kitasato Daigaku

Wasaburo Koizumi

Kitasato University

Research article

Keywords: gastric cancer, Helicobacter pylori, atrophic gastritis, Kita-Kyushu lung cancer antigen-1 
Posted Date: October 9th, 2020

DOl: https://doi.org/10.21203/rs.3.rs-87460/v1

License: (c) (i) This work is licensed under a Creative Commons Attribution 4.0 International License. Read Full License 


\section{Abstract}

Background: Kita-Kyushu lung cancer antigen-1 (KK-LC-1) is a cancer/testis antigen (CTA) and is an attractive target for immunotherapy. An earlier study from our group demonstrated frequent KK-LC-1 expression in gastric cancers (GC) and non-tumor sites of the stomach carrying a tumor. Additionally, there was a correlation to Helicobacter pylori $(\mathrm{Hp})$ infection. Currently it remains unclear whether KK-LC-1 is expressed in stomachs without gastric cancer.

Methods: In the present study, we investigated differences in KK-LC-1 gene expression at non-tumor sites of stomachs with or without a tumor from $118 \mathrm{GC}$ patients and 115 non-GC patients. Fisher's exact test was used for the statistical analyses.

Results: Our results show that KK-LC-1 gene expression was detected in $77 \%$ of non-tumor sites in stomachs with a tumor. Such findings were significantly higher than in stomachs without a tumor $(7 \%, \mathrm{P}$ $<0.0001)$. All patients with KK-LC-1 expression at non-tumor sites of their stomachs without tumors carried $H p$.

Conclusions: KK-LC-1 appears to be detected in the stomach's precancerous condition, but not in an atrophic stomach with Hp. KK-LC-1 may be a useful marker for gastric cancer prediction.

\section{Introduction}

Worldwide, gastric cancer (GC) is the third leading cause of cancer-related death [1]. In Japan, GC has the second highest incidence rate of all cancers. Most cases are caused by Helicobacter pylori $(\mathrm{Hp})$ infection [2]. Detecting and eradicating $H p$ infection may reduce GC risk. Furthermore, ABC diagnosis using an anti$H p$ antibody and measuring the serum levels of pepsinogen $\mathrm{I} / \mathrm{II}$ is an approach used for GC's risk diagnosis [3]. However, this approach has proven to be inaccurate, indicating the occurrence of only 1$5 \%$ of GC in high risk groups. As a consequence, more precise methods are needed for risk diagnosis [4].

To date, numerous tumour-associated antigens have been identified in various human cancers [5]. Among them, cancer/testis antigens (CTAs) are particularly attractive targets for immunotherapy. Such antigens have minimal or no expression in normal tissues, except for germline tissues, while they are aberrantly expressed in a range of human cancers [6]. CTAs may be advantageous targets for systemic cancer diagnosis due to their specific and broad expression patterns in various types of cancer. However, it has been shown that the individual CTA expression rate is insufficient for diagnostic applications [7].

Among the known CTAs, the Kita-Kyushu lung cancer antigen-1 (KK-LC-1), also known as CT83 and Cxorf61, includes epitope peptides recognised by cytotoxic T lymphocytes (CTLs). CTLs against KK-LC-1 predominantly accumulate in tumour-infiltrating lymphocytes. This leads to a good response to adaptive immunotherapy [8]. Except for the testis, KK-LC-1 is not expressed in normal tissues. It is expressed in 33, 82 and $75 \%$ of non-small cell lung cancers, GC and triple negative breast cancers, respectively [9-12]. 
In an earlier study, we reported that $\mathrm{Hp}$ infection induces expression of specific CTAs, in addition to causing malignant transformation of host cells. Additionally, we found that, in clinical GC, there is a correlation between KK-LC-1 expression and Hp infection [13], [14]. These findings suggest a possible correlation between KK-LC-1 expression and $H p$ infection, an early cancer-causing event. Therefore, KKLC-1 could be used as a novel candidate for cancer prediction and diagnosis.

In GC, KK-LC-1 has a 79\% expression rate during the cancer's early stages[15]. Such findings indicate that KK-LC-1 expression occurs at the beginning of a malignancy and is subsequently maintained. Furthermore, our group has observed that KK-LC-1 is expressed in the premalignant lesions of the stomach [16]. However, to date, it remains to be clarified whether the non-premalignant stomach expresses KK-LC-1. In the present study, we clarified the difference in KK-LC-1 expression at non-tumour sites of stomachs with or without a tumour. Additionally, we assessed the clinical utility of KK-LC-1 for the surveillance of GC's occurrence.

\section{Methods}

\section{Patients}

Patients underwent an endoscopic survey of the oesophagus, stomach and duodenum. In cases of atrophic stomach, two portions, one from the lower and one from the middle corpus of the stomach, were sampled and subjected to a rapid urease test (RUT). After RUT confirmation, we collected the gastric specimens. Between March 2016 and August 2017, 412 patients underwent RUT at Kitasato University Medical Centre. Of these patients, a subset $(n=199)$ signed an informed consent after RUT. Each specimen was checked for RNA quality by $\beta$-actin expression. Finally, 115 samples were used in the present study. The Human Ethics Review Committee of Kitasato University Medical Centre, Japan (Approval No. $29-16$ and No.29-18) approved the study protocol. All the experiments were performed in accordance with relevant guidelines and regulations. All patients signed an informed consent prior to resection of the tissue samples used in this study.

Between August 2012 and June 2017, 196 patients underwent surgical resection for GC at the Department of Surgery, Kitasato University Medical Centre, Kitamoto, Japan. We obtained both tumour sample and two or four non-tumour samples, which were located far from tumour site and obtained randomly, from the GC stomach. Based on $\beta$-actin expression levels, all samples had sufficient mRNA quality. Finally, we enrolled $118 \mathrm{GC}$ patients in the present study. Furthermore, to compare RUT specimens, we selected 22 GC patients whose two non-tumour samples were included one from the lower and middle corpuses each. The clinicopathological findings were classified according to the Japanese Classification of Gastric Carcinoma (14th edition) [17].

\section{Tissue specimens}

Following collection, each specimen was immediately stored at $4{ }^{\circ} \mathrm{C}$ overnight in RNAlater (Life Technologies, Carlsbad, CA). Samples were subsequently stored at $-80^{\circ} \mathrm{C}$ until use. For the samples from GC patients, we performed hematoxylin-eosin staining on each adjacent site sampled to assess 
gene expression. The aim was to confirm the predominance of tumour cells in the tumour site and no contamination of tumour cells in the non-tumour site. Furthermore, we planned on distinguishing the sampled non-tumour site into the fundic gland, borderline or pyloric gland.

\section{Expression of CTAs}

A QIACUBE and RNeasy Tissue Mini Kit (Qiagen, Hilden, Germany) were used to isolate total RNA from each sample according to the manufacturer's instructions. Total RNA was then converted to cDNA using oligo $p(d N)_{6}$ random primers and Superscript III reverse transcriptase (Life Technologies). The expression of $\beta$-actin, MAGE-A1, -A3 and -A4, NY-ESO-1, and SSX-4 was measured with TaqMan Gene Expression Assays (IDs: Hs99999903_m1, Hs00607097_m1, H200366532_m1, Hs00365979_m1, Hs00265824_m1, and Hs02341532_m1, respectively). A 7900HT Fast Real-Time PCR System (Life Technologies) was used to perform the analyses. The threshold cycle number of cDNAs converted from RNAs was measured for $\beta$-actin. Real-time PCR was performed in a $20 \mu \mathrm{L}$ reaction containing the following mix: $5 \mu \mathrm{L}$ cDNA template, $10 \mu \mathrm{L}$ FastStart Universal Probe Master Mix (Roche, Mannheim, Germany) and $1 \mu \mathrm{L}$ of TaqMan Gene Expression Assay. Samples were passed with $\leq 30$ threshold cycles $\left(C_{T}\right)$ of $\beta$-actin and then assessed for CTAs expression. The expression of CTAs except for KK-LC-1 were assessed for positive with $\leq 45 \mathrm{C}_{\mathrm{T}}$. KK-LC-1 expression was examined by 40 cycle-end point RT-PCR since an appropriate probe for KK-LC-1 mRNA's detection was unavailable. PCR amplification was performed in $20 \mu \mathrm{L}$ PCR reactions containing the following components: $2 \mu \mathrm{L}$ of cDNA template, rTaq (Takara, Tsu, Japan), dNTPs (Roche,

Basel, Switzerland) and $500 \mathrm{nM}$ each of gene-specific primers ATGAACTTCTATTTACTCCTAGCGAGC and TTAGGTGGATTTCCGGTGAGG (Sigma-Aldrich Japan, Shinagawa, Japan). The annealing temperature was $67^{\circ} \mathrm{C}$. We used 40 cycles to yield a 342-bp product. PCR products were visualised by ethidium bromide staining and ultraviolet light exposure, following electrophoresis on a $1.5 \%$ agarose gels.

\section{Statistical analysis}

Fisher's exact test was used for the statistical analyses between KK-LC-1 expression and each factor. For the analysis, JMP14.0 (SAS institute Japan, Minato-ku, Japan) was used.

\section{Results}

\section{CTAs expression in GC tumour}

To determine the fractions of the tumour site expressing specific CTA genes, reverse transcriptionpolymerase chain reaction (RT-PCR) was used. Of the $118 \mathrm{GC}$ tumours evaluated, 92 specimens (78.0\%) expressed KK-LC-1 (Table 1). We found that other CTAs, specifically Melanoma antigen gene (MAGE)-A1, MAGE-A3, MAGE-A4, New York oesophageal squamous cell carcinoma-1 and Synovial sarcoma, $\mathrm{X}$ breakpoint 4 had the following expressing frequencies: $29.7 \%, 32.2 \%, 19.5 \%, 13.6 \%$ and $17.8 \%$, respectively. 
Table 1 The cancer/testis antigens (CTA )

expression in 118 tumors of gastric cancer

\begin{tabular}{cccc}
\hline CTA & Positive & Negative & $\begin{array}{c}\text { Frequency } \\
(\%)\end{array}$ \\
\hline \hline KK-LC-1 & 92 & 26 & 78.0 \\
MAGE-A1 & 35 & 83 & 29.7 \\
MAGE-A3 & 38 & 80 & 32.2 \\
MAGE-A4 & 23 & 95 & 19.5 \\
NY-ESO-1 & 16 & 102 & 13.6 \\
SSX4 & 21 & 97 & 17.8 \\
\hline
\end{tabular}

KK-LC-1, Kita-Kyushu lung cancer antigen-1;

MAGE, Melanoma antigen gene; NY-ESO-1, New

York esophageal squamous cell carcinoma-1;

SSX4, Synovial sarcoma, X breakpoint 4.

\section{$K K-L C-1$ expression in non-tumour sites of stomach with or without tumour}

Our analysis results showed that for 69 out of 118 (58.5\%) GC patients, KK-LC-1 was detected at one or more non-tumour sites (Table 2). There was no significant difference in the positive rate between the GC patients sampled two and four sites ( $p=0.8517$, Supplementary Table S1). 
Table 2 KK-LC-1 detection at non-tumour sites between GC and Non-GC patients

KK-LC-1 detection

Positive Negative Positive rate (\%)

\begin{tabular}{|c|c|c|c|c|}
\hline GC patients & 69 & 49 & $58.5 \neg$ & \\
\hline L\&M & 17 & 5 & 77.3 & 7 \\
\hline Non-GC patients & 8 & 107 & $7.0 ـ$ & 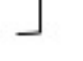 \\
\hline $\mathrm{Hp}(+)$ & 8 & 61 & 11.6 & " \\
\hline $\mathrm{Hp}(-)$ & 0 & 46 & 0.0 & \\
\hline
\end{tabular}

KK-LC-1, Kita-Kyushu lung cancer antigen-1; GC, gastric cancer; L\&M, sampled each lower and middle corpus;

Hp, Helicobacter pylori infection. *, p $<0.0001$.

To focus on the GC patients samples were obtained from lower and middle corpus of the two non-tumour sites as those in non-GC patients, our analysis results showed that for 17 out of the $22(77.3 \%)$ patients, KK-LC-1 was expressed at either of the two or both of the two non-tumour sites and its positive rate was significantly higher than others (45.7\%, $\mathrm{p}=0.0276$, Supplementary Table S2). The non-tumour site of the lower corpus [including one border line (B) and 21 pyloric glands $(P)$ ] had a $68.2 \%$ expression of KK-LC-1 (Supplementary Figure S1). The middle corpus (including seven B, $11 \mathrm{P}$ and four fundic glands) had a $27.3 \%$ expression of KK-LC-1 (Supplementary Figure S1).

On the contrary, in non-GC patients with gastric atrophy, we found eight of 115 (7.0\%) with KK-LC-1 expression. Of note, KK-LC-1's expression rate was significantly lower than that of GC patients $(p<$ 0.0001 , Table 2). All patients in whom KK-LC-1 was detected had Hp infection (Table 2). When considering exclusively $\mathrm{Hp}$-positive non-GC patients, we observed $11.6 \%$ with KK-LC-1 expression ( $\mathrm{p}<$ 0.0001, Table 2). Supplementary Table S3 lists non-GC patients with KK-LC-1 expression. We did not identify specific remarks in the KK-LC-1 positive patients except for patient NGC\#006, who had a mass in the stomach. A biopsy of the mass revealed an adenoma. After two months, the patient underwent an endoscopic surgical dissection against the adenoma. The final pathological diagnosis was adenocarcinoma in adenoma.

\section{Discussion}


KK-LC-1 is a CTA due to its absence of expression in normal tissues, except for the testis. However, it is expressed in cancers of multiple organs [7, 10]. Furthermore, in an earlier study, Fukuyama et al. clarified the specific phenomena at the basis of the correlation between KK-LC-1 expression and Hp infection. Additionally, we previously showed how KK-LC-1 expression was frequently detected at non-tumour sites of the stomach occurring GC $[14,16]$. However, from these studies, it remained unclear whether KK-LC-1 was expressed in the stomach of patients who were not carrying a tumour and with Hp infection. In the present study, we proved that KK-LC-1 was frequently expressed in non-tumour sites of the GC stomach but infrequently in those of non-GC stomach (Fig. 1). Furthermore, we also proved that, while KK-LC-1 expression is correlated with $H p$ infection, it is not exclusively an indicator of Hp infection.

KK-LC-1's expression might become a new indicator for measuring precancerous levels in the stomach. The difference of non-tumour site between GC and non-GC is the precancerous level. Of note, epigenetic alterations were known as precancerous level indicators. In an earlier study, Compare et al. reported that global hypomethylation of the gastric mucosa gradually advanced from $\mathrm{Hp}$-negative gastritis to $\mathrm{Hp}$ positive gastritis, Hp-positive chronic gastritis and gastric carcinoma [18]. In contrast, Leodolter et al. showed that global hypomethylation occurred during the early stages of gastritis, regardless of $H p$ infection [19]. KK-LC-1's expression may help us understand whether the stomach is close to a malignant level, independently from the accumulation of genetic/epigenetic alterations.

KK-LC-1 was not detected at non-tumour sites of the stomachs of three GC patients. However, its expression was detected in tumour sites of the same patients. Such an issue may be resolved by sampling multiple mucosa sites from the lower corpus. Supplementary Figure S1 and results from our previous study show that KK-LC-1's expression rate in the lower corpus was of 68.1 and $66.1 \%$, respectively [16].

We observed one non-GC patient whose non-tumour sites of the stomach expressed KK-LC-1; this patient was later diagnosed with GC. Specifically, following a first pathological observation of a biopsy, an adenoma was identified. However, the sympatric specimen of endoscopic submucosal dissection (ESD) has a diagnosis of adenocarcinoma in adenoma. On the biopsy, RUT was performed and its specimen, allopatric of ESD, expressed KK-LC-1. This phenomenon may indicate that detecting KK-LC-1 marks GC's presence in the stomach's allopatric sites.

Additionally, KK-LC-1 expression was observed in non-tumour sites of GC patients. Of note, GC patients undergoing mucosal resection (including ESD) more frequently presented with metachronous cancers from gastric remnants than during surgical resection[20]. Such findings suggest that KK-LC-1 might represent a prediction marker of GC occurrence. Non-GC patients, in whom KK-LC-1 was detected, are under follow-up to identify possible GC development. However, all non-GC patients with KK-LC-1 received antibiotics against $H p$. Therefore, genetic/epigenetic alterations caused by $H p$ will not accumulate, preventing GC from developing. Nonetheless, GC can occur following Hp eradication [21]. In the event that, in the future, GC occurs in the seven non-GC patients with KK-LC-1, the latter might become a new predictive marker for GC. 


\section{Conclusion}

This study indicated that KK-LC-1 was frequently expressed in non-tumour sites of the GC stomach but infrequently in those of non-GC stomach. In addition, all non-GC patients with KK-LC-1 expression were observed Hp infection. It means that KK-LC-1 appears to be detected in the stomach's precancerous condition, but not in an atrophic stomach with Hp. KK-LC-1 may be a useful marker for gastric cancer prediction.

\section{Abbreviations}

GC, gastric cancer; Hp, Helicobacter pylori; CTA, cancer/testis antigen; KK-LC-1, Kita-Kyushu lung cancer antigen-1; CTL, cytotoxic T lymphocyte; RUT, rapid urease test; RT-PCR, reverse transcription-polymerase chain reaction; MAGE, melanoma antigen gene; B, border line; P, pyloric gland; ESD, endoscopic submucosal dissection

\section{Declarations}

Ethic approval: The study protocol was approved by the Human Ethics Review Committee of Kitasato University Medical Center, Japan (Approval No. 29-16 and No.29-18).

Consent for publication: All patients signed an informed consent prior to resection of the tissue samples used in this study.

Data availability: All data generated or analyzed during this study are available from the corresponding author on reasonable request.

Author contributions: Toshikazu Otsuka: Data curation, Funding acquisition, Formal analysis Writing original draft.: Takashi Fukuyama: Conceptualization, Funding acquisition, Investigation, Methodology, Project administration, Resources, Validation, Writing - review \& editing.: Nobue Futawatari: Conceptualization, Resources.: Kumiko Tahara: Methodology, Resources.: Masaaki Watanabe: Methodology, Resources.: Yoshinobu Ichiki: Conceptualization, Supervision.: Yatsushi Nishi: Resources.: Yoshihito Takahashi: Validation, Visualization.: Hitoshi Yamazaki: Conceptualization, Data curation. : Noritada Kobayashi: Writing - review \& editing.: Mitsuhiro Kida: Supervision.: Hiroshi Nonoguchi: Conceptualization, Data curation.: Wasaburo Koizumi: Supervision.

Funding : T. Otsuka acknowledges support from Grant-in-Aid for research from the Kitasato University Medical Center (H27-030). T. Fukuyama acknowledges support from ALL KITASATO PROJECT STUDY, JSPS KAKENHI (19K09226) and the Takeda Science Foundation.

Competing interests: The authors declare no competing interests.

Conflicts of interest: The authors have no potential conflicts of interest to disclose. 
Acknowledgments: The authors thank Ms. Rui Yamamura, Ms. Tomoko Tajima, Ms. Mami Uchida, and Ms. Shizue Shimamura for their technical assistance and Enago (www.enago.jp) for English language review.

\section{References}

1. Stewart BW WC, editors World Cancer Report 2014 Lyon, France: International Agency for Research on Cancer 2014.

2. Uemura N, Okamoto S, Yamamoto S, Matsumura N, Yamaguchi S, Yamakido M, Taniyama K, Sasaki N, Schlemper RJ: Helicobacter pylori Infection and the Development of Gastric Cancer. New England Journal of Medicine 2001, 345(11):784-789.

3. Miki K: Gastric cancer screening by combined assay for serum anti-Helicobacter pylori lgG antibody and serum pepsinogen levels - "ABC method". Proceedings of the Japan Academy, Series B 2011, 87(7):405-414.

4. Mizuno S, Miki I, Ishida T, Yoshida M, Onoyama M, Azuma T, Habu Y, Inokuchi H, Ozasa K, Miki K et al: Prescreening of a high-risk group for gastric cancer by serologically determined Helicobacter pylori infection and atrophic gastritis. Dig Dis Sci 2010, 55(11):3132-3137.

5. van der Bruggen P, Traversari C, Chomez P, Lurquin C, De Plaen E, Van den Eynde B, Knuth A, Boon T: A gene encoding an antigen recognized by cytolytic $T$ lymphocytes on a human melanoma. Science 1991, 254(5038):1643-1647.

6. Scanlan MJ, Simpson AJ, Old LJ: The cancer/testis genes: review, standardization, and commentary. Cancer immunity 2004, 4:1.

7. Futawatari N, Fukuyama T, Yamamura R, Kobayashi N: Helicobacter Pylori Infection Induces Gastric Cancer and the Cancer/Testis Antigens Expression. J Infect Dis Ther 2017, 5(6):e1000345.

8. Stevanovic S, Pasetto A, Helman SR, Gartner JJ, Prickett TD, Howie B, Robins HS, Robbins PF, Klebanoff CA, Rosenberg SA et al: Landscape of immunogenic tumor antigens in successful immunotherapy of virally induced epithelial cancer. Science 2017, 356(6334):200-205.

9. Shigematsu Y, Hanagiri T, Shiota H, Kuroda K, Baba T, Mizukami M, So T, Ichiki Y, Yasuda M, So T et al: Clinical significance of cancer/testis antigens expression in patients with non-small cell lung cancer. Lung Cancer 2010, 68(1):105-110.

10. Fukuyama T, Hanagiri T, Takenoyama M, Ichiki Y, Mizukami M, So T, Sugaya M, So T, Sugio K, Yasumoto K: Identification of a new cancer/germline gene, KK-LC-1, encoding an antigen recognized by autologous CTL induced on human lung adenocarcinoma. Cancer Res 2006, 66(9):4922-4928.

11. Shida A, Futawatari N, Fukuyama T, Ichiki Y, Takahashi Y, Nishi Y, Kobayashi N, Yamazaki H, Watanabe M: Frequent High Expression of Kita-Kyushu Lung Cancer Antigen-1 (KK-LC-1) in Gastric Cancer. Anticancer research 2015, 35(6):3575-3579.

12. Paret C, Simon P, Vormbrock K, Bender C, Kolsch A, Breitkreuz A, Yildiz O, Omokoko T, Hubich-Rau S, Hartmann $C$ et al: CXorf61 is a target for T cell based immunotherapy of triple-negative breast 
cancer. Oncotarget 2015, 6(28):25356-25367.

13. Fukuyama T, Yamazaki T, Fujita T, Uematsu T, Ichiki Y, Kaneko H, Suzuki T, Kobayashi N: Helicobacter pylori, a carcinogen, induces the expression of melanoma antigen-encoding gene (Mage)-A3, a cancer/testis antigen. Tumour Biol 2012, 33(6):1881-1887.

14. Fukuyama T, Futawatari N, Ichiki Y, Shida A, Yamazaki T, Nishi Y, Nonoguchi H, Takahashi Y, Yamazaki H, Kobayashi N: Correlation Between Expression of the Cancer/Testis Antigen KK-LC-1 and Helicobacter pylori Infection in Gastric Cancer. In vivo (Athens, Greece) 2017, 31(3):403-407.

15. Futawatari N, Fukuyama T, Yamamura R, Shida A, Takahashi Y, Nishi Y, Ichiki Y, Kobayashi N, Yamazaki H, Watanabe M: Early gastric cancer frequently has high expression of KK-LC-1, a cancertestis antigen. World J Gastroenterol 2017, 23(46):8200-8206.

16. Fukuyama T, Futawatari N, Yamamura R, Yamazaki T, Ichiki Y, Ema A, Ushiku H, Nishi Y, Takahashi Y, Otsuka T et al: Expression of KK-LC-1, a cancer/testis antigen, at non-tumour sites of the stomach carrying a tumour. Scientific reports 2018, 8(1):6131.

17. Japanese classification of gastric carcinoma: 3rd English edition. Gastric Cancer 2011, 14(2):101112.

18. Compare D, Rocco A, Liguori E, D'Armiento FP, Persico G, Masone S, Coppola-Bottazzi E, Suriani R, Romano M, Nardone G: Global DNA hypomethylation is an early event in Helicobacter pylori-related gastric carcinogenesis. J Clin Pathol 2011, 64(8):677-682.

19. Leodolter A, Alonso S, Gonzalez B, Pa Ebert M, Vieth M, Röcken C, Wex T, Peitz U, Malfertheiner P, Perucho M: Somatic DNA Hypomethylation in H. pylori-Associated High-Risk Gastritis and Gastric Cancer: Enhanced Somatic Hypomethylation Associates with Advanced Stage Cancer, vol. 6; 2015.

20. Kobayashi M, Narisawa R, Sato Y, Takeuchi M, Aoyagi Y: Self-limiting risk of metachronous gastric cancers after endoscopic resection. Digestive endoscopy : official journal of the Japan Gastroenterological Endoscopy Society 2010, 22(3):169-173.

21. Fukase K, Kato M, Kikuchi S, Inoue K, Uemura N, Okamoto S, Terao S, Amagai K, Hayashi S, Asaka M: Effect of eradication of Helicobacter pylori on incidence of metachronous gastric carcinoma after endoscopic resection of early gastric cancer: an open-label, randomised controlled trial. The Lancet 2008, 372(9636):392-397.

\section{Figures}




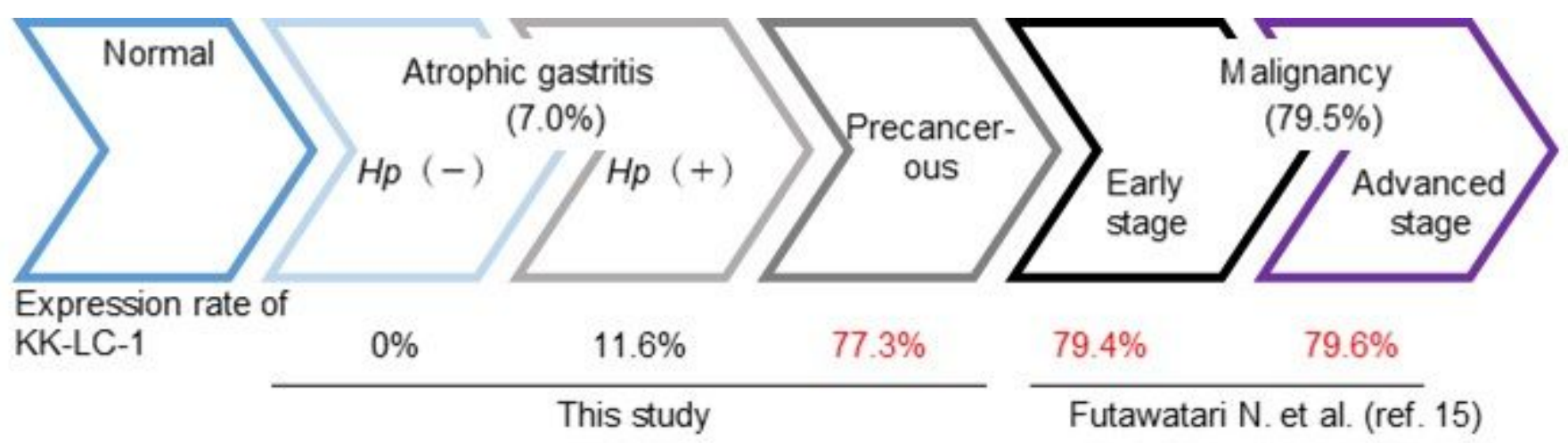

\section{Figure 1}

The expression rate of KK-LC-1 among the transition of gastric abnormality The expression rate of KK-LC1 was summarised for each stage of gastric abnormality. It is consistently known that KK-LC-1 is not expressed in the stomach under the normal condition. However, according to the estimation of abnormality-indicating factors, the KK-LC-1 expression rate increased in this order: atrophic gastritis without and with $\mathrm{Hp}$ infection, non-tumour sites of precancerous stomach with gastric cancer and early and advanced gastric cancer tumours. This study proved that KK-LC-1 expression is clearly discrepant between noncancerous and cancerous stomach regardless of Hp infection. KK-LC-1 expression rate in malignancy including early and progressive stages was adopted from Futawatari et al [15]. Hp: Helicobacter pylori infection; -: Hp negative; +: Hp positive.

\section{Supplementary Files}

This is a list of supplementary files associated with this preprint. Click to download.

- Additionalfile1.docx 